\title{
A Study on Community Acquired Bloodstream Infections and Molecular Characterization of Resistant Pathogens in a Tertiary Care Hospital
}

\author{
A. Priyadharshini and C.P. Ramani* \\ Institute of Microbiology, Madras Medical College, Chennai, India \\ *Corresponding author
}

\begin{abstract}
Keywords
Communityacquired sepsis, Bloodstream infections, Blood culture, ESBL producers, MRSA, PCR

Article Info

Accepted:

18 January 2019

Available Online:

10 February 2019 association with any prior medical interventions. Aims of the study are to identify the clinical profile of patients, detect the pathogens causing community-acquired bloodstream infections (CA-BSI) and their antimicrobial susceptibility pattern and to perform the molecular characterization of resistant pathogens. Under strict aseptic precautions, blood samples were collected and processed as per standard protocol and isolates identified. Their antimicrobial susceptibility testing was performed by Kirby-Bauer disk diffusion method under CLSI guidelines. Vancomycin sensitivity tested using Vancomycin Screen agar and confirmed by E-strip test. Resistant strains were characterized by PCR. Blood culture in 150 patients, detected 12 patients (8\%) with CA-BSI. Gram-positive organisms 58\% (MSSA $85.7 \%$ and $14.3 \%$ MRSA) isolated, were highly sensitive to Erythromycin, Vancomycin, Linezolid and 42\% Gram-negative organisms (Escherichia coli 60\% which were ESBL producers, 20\% Acinetobacter baumannii and 20\% Pseudomonas aeruginosa) isolated, were highly sensitive to Amikacin, Tetracycline each $100 \%$ respectively. bla $T E M$ and bla CTX-M genes among ESBL producers and $m e c A$ gene in MRSA isolate were positive by PCR. CA-BSI are rising as a major health problem in the upcoming years due to the emergence of antimicrobial resistant strains in the community as well, like ESBL producers, MRSA, etc. Hence, proper surveillance, the framing of appropriate antibiotic policy and preventive strategies curtails the spread of these resistant strains in the community.
\end{abstract}

\section{A B S T R A C T}

Community-acquired bloodstream infections are the infections detected within 48 hours of hospitalization, showing positive blood culture and develop spontaneously without an

\section{Introduction}

Bloodstream infections are one of the serious and life-threatening clinical conditions leading to deleterious consequences with a mortality rate ranging from 20-40 \%.1, 2 Hence, needs immediate attention and treatment. Advances in blood culture techniques have resulted in efficient and reliable methodologies for the detection of causative pathogens. Bloodstream infections are classified traditionally as nosocomial and community-acquired bloodstream infections. ${ }^{\mathbf{3 , 4}}$ Community-acquired bloodstream infections refers to the infections detected within 48 hours of hospitalization, showing positive blood culture and develops spontaneously without an association with any prior medical 
interventions. $^{5} \quad$ Community-acquired bloodstream infections are becoming a major health problem in the upcoming years due to the emergence of antimicrobial resistant organisms in community settings as causative agents like, ESBL producing Enterobacteriaceae, Methicillin-resistant Staphylococcus aureus. ${ }^{6}$ Antimicrobial resistant strains once confined to hospital settings are now a potential threat in the community too. Rapid detection of antimicrobial-resistant strain is highly essential, as they are associated with increased mortality and morbidity and due to their high propensity to spread and able to cause a serious threat to public health concern. Phenotypic characterization of microorganisms helps in identification of causative agents of infectious diseases. Molecular characterization of resistant pathogens aids in tracking the spread of antimicrobial resistance in community and hospital settings.

To identify the clinical profile of patients, detect the pathogens causing communityacquired bloodstream infections and their antimicrobial susceptibility pattern and to perform the molecular characterization of resistant pathogens.

\section{Materials and Methods}

Ethical clearance was obtained from the Institutional Ethics Committee before starting the study. This is a cross-sectional study done for a period of 1 year (from March 2017February 2018) at tertiary care centre, chennai, where blood samples from 150 febrile adult patients with suspected sepsis admitted within 48hrs in Medicine wards, Intensive Care Unit and Surgical wards were collected under strict aseptic precautions and were processed as per standard protocol. The isolates were identified based on Gram stain, colony morphology, and various biochemical reactions. Antimicrobial susceptibility testing for isolated organisms done on Mueller Hinton agar plate by Kirby- Bauer disk diffusion method under CLSI guidelines. Using the differential disk, Cefoxitin $(30 \mu \mathrm{g})$, Staphylococcus aureus isolates were categorized into methicillin sensitive and methicillin-resistant strains. ${ }^{7}$

\begin{tabular}{|l|l|l|l|}
\hline Cefoxitin $(30 \mu \mathrm{g})$ & Susceptible & Intermediate & Resistant \\
\hline Zone size & $>=22 \mathrm{~mm}$ & - & $\begin{array}{l}<\text { or } \\
=21 \mathrm{~mm}\end{array}$ \\
\hline
\end{tabular}

Vancomycin sensitivity was tested using Vancomycin screen agar (BHI agar with $6 \mu \mathrm{g} / \mathrm{ml}$ of Vancomycin), where $10 \mu \mathrm{l}$ of bacterial suspension was spot inoculated onto this media and incubated overnight at $37^{\circ} \mathrm{C}$ along with appropriate controls. ${ }^{7}$ After $24 \mathrm{hrs}$ of incubation, the sensitivity pattern was interpreted as follows-

-If no visible growth at spot inoculated sitereported as sensitive to Vancomycin.

-If visible growth ( $>1$ colony) at spot inoculated site was present -reported as resistant to Vancomycin.

\section{E-test procedure}

Using an inoculating loop, 4-5 isolated colonies of Staphylococcus were transferred to a test tube containing peptone water and emulsified. Incubated it for 2-4hrs until the growth equal to a $0.5 \mathrm{McF}$ arland turbidity standard was reached. A sterile cotton swab was dipped into this inoculum suspension and pressed against the inside wall of the tube to remove excess fluid and then streaked over the entire surface of Mueller Hinton agar plate evenly in three directions. The surface of agar was allowed to dry completely, and then an Estrip was applied to the agar surface with the MIC scale facing upwards. The plate was then incubated at $37^{\circ} \mathrm{C}$ for overnight incubation. After 24 hrs of incubation, the MIC value was read at a point where the edge of inhibition ellipse intersects the strip. 


\begin{tabular}{|l|l|l|l|}
\hline Vancomycin & Susceptible & Intermediate & Resistant \\
\hline $\mathrm{MIC}(\mu \mathrm{g} / \mathrm{ml})$ & $<$ or $=2$ & $4-8$ & $>=16$ \\
\hline
\end{tabular}

Among the Gram-negative organisms identified, ESBL producers detected as follows-

An initial screening test is done by disk diffusion method under CLSI guidelines using Cefotaxime $(30 \mu \mathrm{g})$ disk and Ceftazidime $(30 \mu \mathrm{g})$ disk which was applied on to Mueller Hinton agar plate inoculated with the test organism and incubated at $37^{\circ} \mathrm{C}$ for $24 \mathrm{hrs}$. Screening test denoted ESBL production if zone size was as follows-

\begin{tabular}{|l|l|}
\hline Cefotaxime $(30 \mu \mathrm{g})$ & $<$ or $=27 \mathrm{~mm}$ \\
\hline Ceftazidime $(30 \mu \mathrm{g})$ & $<$ or $=22 \mathrm{~mm}$ \\
\hline
\end{tabular}

The phenotypic confirmatory test is done by disk diffusion method under CLSI guidelines by the combination disk test method using cefotaxime $(30 \mu \mathrm{g})$ disk and cefotaximeclavulanic acid $(30 \mu \mathrm{g} / 10 \mu \mathrm{g})$.

\section{Combination disk test}

Disks containing cephalosporin alone and in combination with clavulanic acid were applied onto Mueller Hinton agar plate inoculated with test organism and incubated at $37^{\circ} \mathrm{C}$ for $24 \mathrm{hrs}$.

\section{Molecular methods}

\section{Characterization of resistant bacterial isolates}

The polymerase chain reaction was performed to detect the resistant genes. It included the following steps -

1. Extraction of DNA from all resistant isolates done using PureFast ${ }^{\circledR}$ Bacterial DNA minispin purification kit
2. PCR amplification of DNA -using following components Master mix $(2 \mathrm{U}$ of Taq DNA polymerase, 10X Taq reaction buffer, $2 \mathrm{mM} \mathrm{MgCl} 2,1 \mu \mathrm{l}$ of $10 \mathrm{mM}$ dNTPs mix and Red Dye PCR additives)-10 $\mu 1$, primer mix (blaTEM gene Primer mix-260bp, blaCTX-M gene Primer mix-295bp, mecA gene Primer mix-220bp)-5 $\mu 1$ and extracted purified DNA-5 $\mu 1$

The PCR products were analyzed using agarose gel electrophoresis, and the sizes of the PCR products were determined by comparing with the DNA ladder ranging from 100bp lower range till 1500bp higher range.

\section{Results and Discussion}

The study group included 150 patients in the age group $>18 y$ rs with clinical suspicion of sepsis admitted within 48hrs in Medical, Surgical wards and Intensive Care Units. Blood culture performed in 150 patients, detected 12 patients (8\%) with communityacquired bloodstream infection. The majority (n-150) presented with fever predominantly followed by next common presentations were cough/dyspnoea, abdominal pain/ vomiting, dysuria, bleeding disorders/Malena (Fig. 1).

Both Gram-positive and Gram-negative organisms were isolated. 58\% of Grampositive organisms were isolated which included [Methicillin sensitive Staphylococcus aureus (MSSA) $85.7 \%$ and 14.3\% Methicillin-resistant Staphylococcus aureus (MRSA)]. $42 \%$ of Gram-negative organisms were isolated which included [Escherichia coli 60\% which were ESBL producers, 20\% Acinetobacter baumannii and 20\% Pseudomonas aeruginosa] (Tables 1 and 2). Gram-positive organisms were found highly sensitive to Erythromycin, Vancomycin and Linezolid each 100\% 
respectively (Table 3). MSSA was found to be highly resistant to Cotrimoxazole $(66.7 \%)$ followed by Ciprofloxacin (50\%). MRSA was found to be highly resistant to Cotrimoxazole, Tetracycline, and Penicillin each 100\% respectively. Gram-negative organisms were found highly sensitive to Amikacin, Tetracycline and Imipenem each 100\% respectively (Table 4). Escherichia coli showed a high level of resistance to Ceftazidime, Cotrimoxazole, Cefotaxime, Ampicillin, and Ciprofloxacin each 100\% respectively. Pseudomonas aeruginosa exhibited a high level of resistance to Ceftazidime $(100 \%)$.
Acinetobacter baumannii was also found resistant to Ceftazidime, Ciprofloxacin, Cotrimoxazole, Gentamicin each 100\% respectively. Percentage of resistant strains among Gram-positive organisms constituted about 14.3\%, and among Gram-negative organisms, the percentage of resistant strains identified was about 60\%. bla TEM and bla $C T X-M$ genes were positive among ESBL (Extended Spectrum Beta-lactamase) producing $E$. coli isolates and mecA gene positive in MRSA (Methicillin-Resistant Staphylococcus aureus) isolate, by PCR (polymerase chain reaction) (Table 5 and Fig. 2).

Table.1 Gram-positive organisms

\begin{tabular}{|l|c|c|}
\hline Organisms & No. Isolated & Percent \\
\hline Staphylococcus aureus (MSSA) & 6 & 85.7 \\
\hline Staphylococcus aureus (MRSA) & 1 & 14.3 \\
\hline Total & 7 & 100 \\
\hline
\end{tabular}

Table.2 Gram negative organisms

\begin{tabular}{|l|c|c|}
\hline Organisms & No. Isolated & Percent \\
\hline E. coli & 3 & 60 \\
\hline Pseudomonas aeruginosa & 1 & 20 \\
\hline Acinetobacter baumannii & 1 & 20 \\
\hline Total & 5 & 100 \\
\hline
\end{tabular}


Table.3 Antimicrobial susceptibility pattern among Gram positive organisms

\begin{tabular}{|l|l|l|l|l|}
\hline Organism & $\begin{array}{l}\text { Methicillin } \\
\text { Staphylococcus }\end{array}$ & $\begin{array}{r}\text { Sensitive } \\
\text { (MSSA) }\end{array}$ & $\begin{array}{l}\text { Methicillin } \\
\text { Staphylococcus } \\
\text { (MRSA) }\end{array}$ & $\begin{array}{r}\text { Resistant } \\
\text { aureus }\end{array}$ \\
\hline Number Isolated & 6 & $\mathbf{R}(\%)$ & 1 & S $(\%)$ \\
\hline Drugs & S $(\%)$ & 50 & 100 & 0 \\
\hline Ciprofloxacin & 50 & 0 & 0 & 100 \\
\hline Penicillin & 100 & 66.7 & 0 & 100 \\
\hline Cotrimoxazole & 33.3 & 0 & 100 & 0 \\
\hline Erythromycin & 100 & 0 & 100 & 0 \\
\hline Linezolid & 100 & 0 & 0 & 100 \\
\hline Tetracycline & 100 & 0 & 100 & 0 \\
\hline Vancomycin & 100 & & & \\
\hline
\end{tabular}

Table.4 Antimicrobial susceptibility pattern of Gram negative organisms

\begin{tabular}{|c|c|c|c|c|c|c|}
\hline Organism & \multicolumn{2}{|c|}{ Escherichia coli } & \multicolumn{2}{|c|}{$\begin{array}{l}\text { Pseudomonas } \\
\text { aeruginosa }\end{array}$} & \multicolumn{2}{|c|}{$\begin{array}{l}\text { Acinetobacter } \\
\text { baumannii }\end{array}$} \\
\hline No. Isolated & \multicolumn{2}{|l|}{3} & \multicolumn{2}{|c|}{1} & \multicolumn{2}{|c|}{1} \\
\hline Drugs & S (\%) & $\mathbf{R}(\%)$ & $\mathbf{S}(\%)$ & $\mathbf{R}(\%)$ & $\mathrm{S}(\%)$ & $\mathbf{R}(\%)$ \\
\hline Amikacin & 100 & 0 & 100 & 0 & 0 & 100 \\
\hline Gentamicin & 33.3 & 66.7 & 100 & 0 & 0 & 100 \\
\hline Ciprofloxacin & 0 & 100 & 100 & 0 & 0 & 100 \\
\hline Cotrimoxazole & 0 & 100 & \multicolumn{2}{|l|}{-------- } & 0 & 100 \\
\hline Ampicillin & 0 & 100 & \multicolumn{2}{|l|}{------- } & \multicolumn{2}{|l|}{------- } \\
\hline Cefotaxime & 0 & 100 & \multicolumn{2}{|l|}{ |------- } & \multicolumn{2}{|c|}{------- } \\
\hline $\begin{array}{l}\text { Cefotaxime- } \\
\text { clavulanic acid }\end{array}$ & 100 & 0 & \multicolumn{2}{|l|}{------- } & \multicolumn{2}{|l|}{------- } \\
\hline Ceftazidime & 0 & 100 & 0 & 100 & 0 & 100 \\
\hline Tetracycline & 100 & 0 & \multicolumn{2}{|l|}{------- } & 100 & 0 \\
\hline $\begin{array}{l}\text { Piperacillin- } \\
\text { Tazobactam }\end{array}$ & \multicolumn{2}{|c|}{---------- } & 100 & 0 & 100 & 0 \\
\hline Imipenem & \multicolumn{2}{|c|}{---------- } & 100 & 0 & 100 & 0 \\
\hline
\end{tabular}

Table.5 Molecular identification of antimicrobial resistant genes by PCR

\begin{tabular}{|l|l|l|}
\hline Resistant strains & Primers & Result \\
\hline ESBL Producers(3) & blaTEM & POSITIVE \\
\hline ESBL Producers(3) & blaCTX-M & POSITIVE \\
\hline MRSA Strain(1) & mecA & POSITIVE \\
\hline
\end{tabular}


Fig.1 Clinical profile of patients with suspected sepsis $(\mathrm{N}=150)$

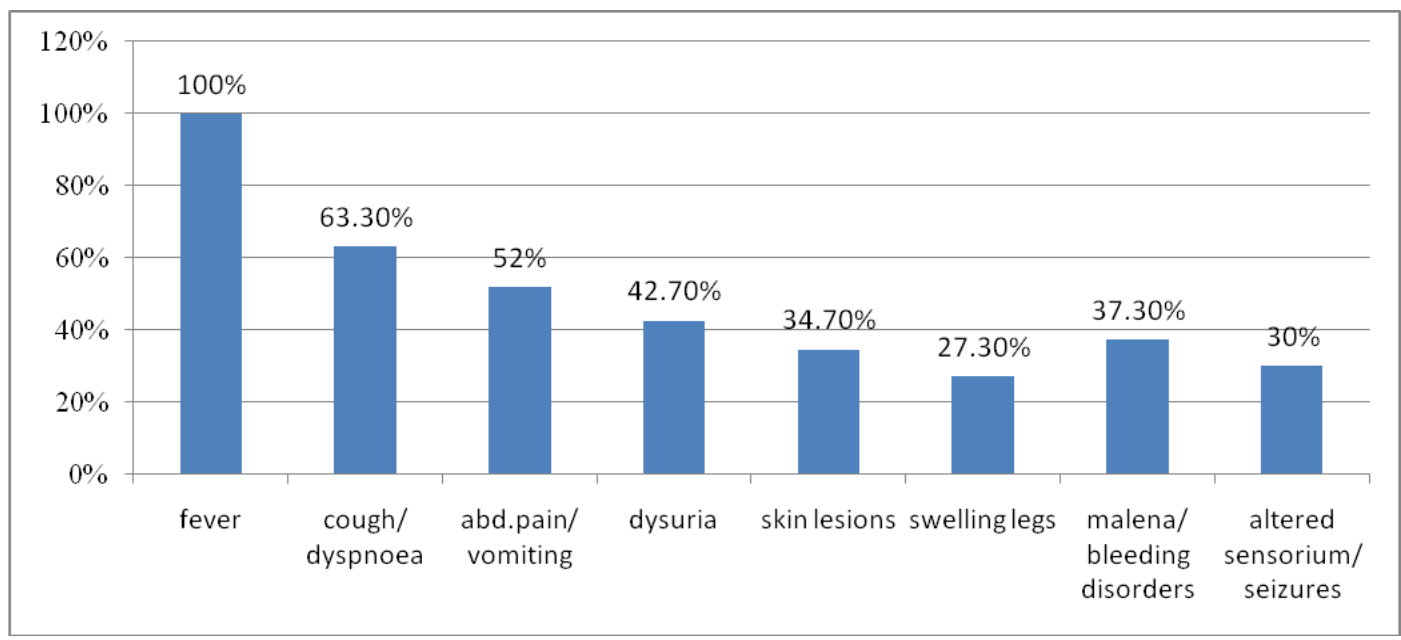

Fig.2 1. bla CTX-M-ladder 2. bla TEM-LADDER 3. NTC-Ladder-mecA
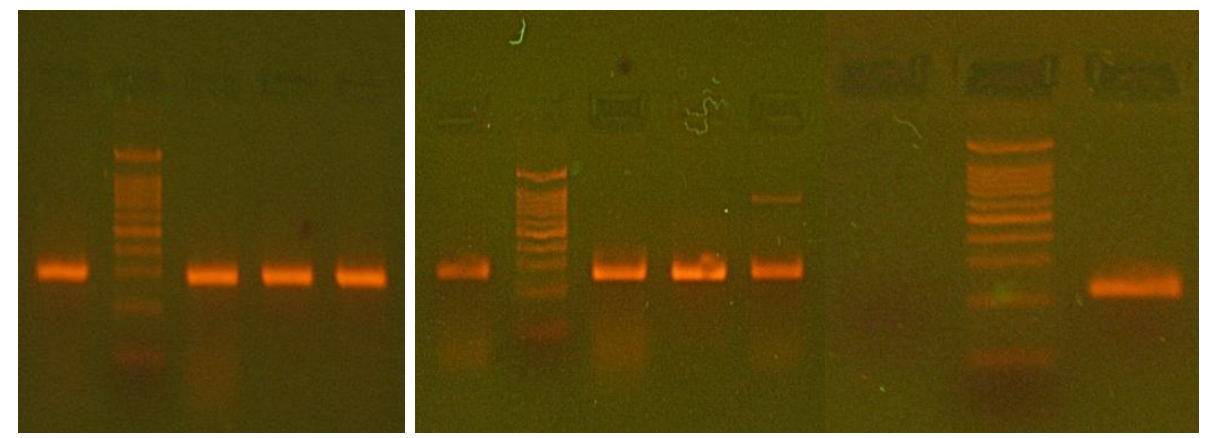

Bloodstream infections are an important cause of mortality and also morbidity related to sepsis. This study was focussed on knowing the burden of community-acquired bloodstream infections in our settings and the pathogens responsible for it.

During the study period of 1 year from March 2017- February 2018, blood culture was done in 150 patients with clinical suspicion of sepsis within $48 \mathrm{hrs}$ of hospital admission. Out of which, community-acquired bloodstream infection was detected in 12 patients $(8 \%)$, in this study. Tufail Soomro et al., ${ }^{\mathbf{8}}$ (2016) concluded in their study that the frequency and incidence of community-acquired bloodstream infection was $7.6 \%$. Sigauque $e t$ $a l .,{ }^{9}$ in their study had identified community- acquired bloodstream infection in $8 \%$ of patients on hospital admission correlating well with our study. In a cohort study of 3901 patients with community-acquired sepsis conducted by Nathan I. Shapiro et al., ${ }^{\mathbf{1 0}}$ the incidence of bloodstream infection at hospital admission was $8.2 \%$.

In the present study, out of 12 patients with community-acquired bloodstream infection, the frequency and distribution of pathogens were 58\% Gram-positive organisms and $42 \%$ Gram-negative organisms.

Among the Gram-positive organisms, 85.7\% were methicillin-sensitive Staphylococcus aureus (MSSA), and $14.3 \%$ were methicillinresistant Staphylococcus aureus (MRSA). 
Hence, among the Gram-positive organisms, $14.3 \%$ were found to be resistant pathogens. In the study conducted by Goncalves- Pereira et al., ${ }^{11}$ also the predominant Gram-positive organism isolated were methicillin-sensitive Staphylococcus aureus and the predominant Gram-negative organisms identified were Escherichia coli. In a study done by Klevens et al., ${ }^{\mathbf{1 2}}$ incidences of community-associated methicillin resistant Staphylococcus aureus infection was found to be $14 \%$.

In this study, among the Gram-negative organisms, Escherichia coli contributed 60\%, Pseudomonas aeruginosa and Acinetobacter baumannii each contributed $20 \%$ respectively. The study by Parkins MD et al., ${ }^{13}$ also showed that the incidence of communityacquired bloodstream infection cases caused by Pseudomonas aeruginosa were $21 \%$, well correlates with our study. Also, in a study of Chung-Ting Chen et al., ${ }^{14}$ (2017), Acinetobacter baumannii isolates were identified as the causatives of communityacquired bloodstream infections and for these isolates, respiratory tract was the primary source involved which matches with the present study where the Acinetobacter baumannii isolate identified was acquired from respiratory tract as primary source of infection. Among the Gram-negative organisms isolated, $60 \%$ were found to be resistant pathogens especially, extendedspectrum beta-lactamase (ESBL) producers among Escherichia coli organisms. Quan et al., ${ }^{15}$ (2017) study revealed $56 \%$ of ESBL producing $E$. coli isolates were identified in community-acquired bloodstream infections.

In the present study, among Gram-positive organisms isolated, methicillin-sensitive Staphylococcus aureus were highly sensitive to Penicillin (100\%), Erythromycin (100\%), Tetracycline (100\%), Linezolid (100\%), Vancomycin (100\%) and were resistant to Cotrimoxazole (66.7\%) and Ciprofloxacin
(50\%). Methicillin-resistant Staphylococcus aureus was highly sensitive to Ciprofloxacin $(100 \%)$, Erythromycin (100\%), Linezolid (100\%), Vancomycin (100\%) and was highly resistant to Penicillin (100\%), Cotrimoxazole $(100 \%)$ and Tetracycline $(100 \%)$.

Among the Gram-negative organisms isolated, Escherichia coli isolates were highly sensitive to Amikacin (100\%), Tetracycline $(100 \%)$ and were found highly resistant to Ciprofloxacin, Cotrimoxazole, Ampicillin, Cefotaxime each $100 \%$ respectively and Gentamicin (66.7\%). Pseudomonas aeruginosa was highly sensitive to Amikacin (100\%), Gentamicin (100\%), Ciprofloxacin (100\%), Piperacillin-Tazobactam (100\%), Imipenem (100\%) and were highly resistant to Ceftazidime (100\%). Acinetobacter baumannii isolate was highly sensitive to Tetracycline (100\%), Piperacillin-Tazobactam $(100 \%)$, Imipenem $(100 \%)$ and were highly resistant to Amikacin (100\%), Gentamicin (100\%), Ciprofloxacin (100\%), Cotrimoxazole $(100 \%)$ and Ceftazidime (100\%). Molecular characterization of resistant isolates was done using polymerase chain reaction (PCR) which showed the presence of bla TEM and bla CTX-M genes, that confirmed ESBL producers among the Escherichia coli isolates and similarly, the presence of mecA gene confirmed methicillin-resistant Staphylococcus aureus. Luzzaro et al., ${ }^{16}$ in his study found that the most prevalent ESBL producing Gramnegative organism was found to be Escherichia coli and TEM- type ESBLs were found to be the most prevalent enzymes $(45.4 \%)$. According to the study by Rossolini et al., ${ }^{17}$ the CTX-M-type ESBLs had undergone a rapid and global spread in Enterobacteriaceae recently. In Mario Tumbarello et al., ${ }^{18}$ study, the predominantly isolated ESBL genes were bla CTX-M (36.5\%) followed by bla TEM gene $(28.7 \%)$. Nagat Sobhy et al., ${ }^{19}$ study emphasized that 
the identification of the mecA gene is the most reliable method for detecting the MRSA isolate.

In conclusion, community-acquired bloodstream infections are rising as a major health problem in upcoming years due to the emergence of antimicrobial resistant organisms which were once confined to hospital settings are now a potential threat in the community settings as well like ESBL producing Enterobacteriaceae, MRSA, etc. Hence, these antimicrobial resistant strains should be promptly identified through proper surveillance. Molecular characterization of resistant pathogens helps in tracking the spread of antimicrobial resistance in the community. Also, appropriate antibiotic policy and preventive strategies have to be framed to curtail the spread of these antimicrobial resistant strains in the community settings.

Funding: Indian Council of Medical Research (ICMR)

\section{Acknowledgement}

I sincerely thank ICMR for extending financial support for this study.

\section{References}

1. Pedersen G, Schonheyder H.C., Sorensen H. T. Source of infection and other factors associated with case fatality in community- acquired bacteremia- a Danish populationbased cohort study from 1992 to 1997. Clin Microbiol Infect 2003; 9:793802.

2. Larry G. Reimer, Michael L. Wilson, Melvin P. Weinstein. Update on detection of bacteremia and fungemia. Clinical Microbiology Reviews, 1997, 10:444-465.
3. J. Rodriguez- Bano, M.D. LopezPrieto, M.M. Portillo, et al., Epidemiology and clinical features of community- acquired, healthcareassociated and nosocomial bloodstream infections in tertiary-care and community hospitals. Clin Microbiol Infect 2010; 16: 1408-1413.

4. Garner JS, Jarvis WR, Emori TG, et al., CDC definitions for nosocomial infections. Am J Infect Control 1988; 16:128-40.

5. Yardena Siegman - Igra, Boaz Fourer, Ruth Orni- Wasserlauf, et al., Reappraisal of community- acquired bacteremia: A proposal of a New Classification for the spectrum of acquisition of bacteremia. Clinical infectious diseases 2002; 34:1431-9.

6. Johann D.D. Pitout, Patrice Nordmann, Kevin B. Laupland et al., Emergence of Enterobacteriaceae producing extended- spectrum $\beta$ lactamases (ESBL) in the community. J antimicro chemo. 2005; 56: 52-59.

7. Clinical and Laboratory Standards Institute (CLSI) M100 27 $7^{\text {th }}$ edition Performance Standards for antimicrobial susceptibility testing.

8. Tufail Soomro, Shiyam Sunder Tikmani, Sumera Aziz Ali. Frequency and Etiology of community- acquired bloodstream infection in hospitalized febrile children. J Med Diagn Meth 2016; 5:217.

9. Sigauque B, Roca A, Mandomandol, et al., Community-acquired bacteremia among children admitted to a rural hospital in Mozambique. Pediatr Infect Dis J. 2009; 28:108-13.

10. Nathan I. Shapiro, Richard E. Wolfe, Sharon B. Wright, et al., Who needs a blood culture? A prospectively derived and validated prediction rule. J Emerg Med 2008; 35:255-264.

11. J. Goncalves Pereira, P.R. Povoa, C. 
Lobo, et al., Bloodstream infections as a marker of community-acquired sepsis severity. Results from the Portuguese Community-acquired sepsis study (SACiUCI study). Clin Microbio Infect 2013; 19:242-248.

12. Klevens RM, Morrison MA, Nadle J, et al., Invasive Methicillin resistant Staphylococcus aureus infections in the United States. JAMA 2007; 298:1763-1771.

13. Parkins MD, Gregson DB, Pitout JD, et al., Population-based study of the epidemiology and the risk factors for Pseudomonas aeruginosa bloodstream infection. Infection 2010; 38:25-32.

14. Chung-Ting Chen, Yung-Chih Wang, Shu-Chen Kuo, et al., Communityacquired bloodstream infections caused by Acinetobacter baumannii; A matched case-control study. j. jmii.2017; 02.004.

15. Quan J, Zhao D, Liu L, et al., High prevalence of ESBL- producing Escherichia coli and Klebsiella pneumoniae in community-onset bloodstream infections in China. J Antimicrob Chemother 2017; 72:273280.
16. Luzzaro F, M. Mezzatesta, C. Mugnaioli, et al., Trends in production of extended spectrum beta-lactamases among Enterobacteria of medical interest: report of the second Italian nationwide survey. J. Clin. Microbiol. 2006; 44: 1659-1664.

17. Rossolini G.M, M.M.D' Andrea, C.Mugnaioli. The spread of CTX-MType extended spectrum $\beta$-lactamases. CLIN. Microbiol. Infect. 2008; 14(suppl.1): S33-S41.

18. Mario Tumbarello, Michela Sali, Enrico Maria Trecarichi, et al., Bloodstream infections caused by Extended- spectrum- $\beta$-lactamaseproducing Escherichia coli: Risk factors for inadequate initial antimicrobial therapy. J Antimicrob Chemother. 2008; 52:3244-3252.

19. Nagat Sobhy, Fatma Aly, Ola Abd El Kader et al., Community-acquired methicillin-resistant Staphylococcus aureus from skin and soft tissue infections(in a sample of Egyptian population): analysis of mecA gene and Staphylococcal cassette chromosome. Braz J Infect Dis.2012; 16:426-431.

\section{How to cite this article:}

Priyadharshini, A and Ramani, C.P. 2019. A Study on Community Acquired Bloodstream Infections and Molecular Characterization of Resistant Pathogens in a Tertiary Care Hospital. Int.J.Curr.Microbiol.App.Sci. 8(02): 2347-2355. doi: https://doi.org/10.20546/ijcmas.2019.802.273 